\title{
Assessing physical and respiratory distress in children with bronchiolitis admitted to a community hospital emergency department: A retrospective chart review
}

\author{
Mika L. Nonoyama, RRT, PhD ${ }^{1,2,3}$, Vinay Kukreti, MD4, Efrosini Papaconstantinou, RN, PhD ${ }^{1,5}$, Rayona Raymond D'cruz, BSc ${ }^{1}$
}

\begin{abstract}
ML Nonoyama, V Kukreti, E Papaconstantinou, R Raymond D'cruz. Assessing physical and respiratory distress in children with bronchiolitis admitted to a community hospital emergency department: A retrospective chart review. Can J Respir Ther 2019;55:16-20. doi: 10.29390/ cjrt-2018-021.

Introduction: Bronchiolitis is a leading cause of infant hospitalization with wide variation in its diagnosis and management, especially in smaller community hospitals. The objective of this study is to describe children admitted to a community-based hospital emergency department (ED) for bronchiolitis and explore alternate assessments of illness severity.

Methods: A retrospective chart review (January to September 2014) of 100 children, < 2 years old and meeting International Classification of Diseases 10 for bronchiolitis. Outcomes included demographics, symptoms, and interventions. In addition, the Respiratory Distress Assessment Instrument (RDAI) score was calculated using documented assessments of wheezing and retractions. Descriptive and comparative statistics were completed with $p<0.05$ considered significant.

Results: The mean (standard deviation) age 10.6 (8.4) months, $n=41$ females. Sixty-seven percent had a chest X-ray (CXR), 17\% oral antibiotics, $65 \%$ bronchodilators, and 19\% oral steroids; $19 \%$ were admitted in hospital. There was a significant difference in RDAI score between those given oral antibiotics (mean (95\% CI), $6.35(4.96-7.75))$ versus not $(4.70(4.20-5.20)), p=0.01$. Those who received a CXR had a significantly higher oxygen flowrate (1.4 (0.6-2.1) litres per minute (lpm)) and worse physical appearance (tri-pod position, head bobbing) versus those who did not $(0.15$ ( -0.05 to 0.35$) \mathrm{lpm})$, $p=0.002$ and $p=0.04$, respectively.

Conclusions: A large number of children admitted to a community-based ED for bronchiolitis received unnecessary CXR and medications. Assessing physical and respiratory distress may be more effective at determining illness severity compared with radiological or laboratory testing. Local clinical practice guidelines may aid in optimal management of bronchiolitis for community-based EDs.
\end{abstract}

Key Words: bronchiolitis; emergency service hospital; retrospective studies; hospitals; community

\section{INTRODUCTION}

Infants who present to the emergency department (ED) with respiratory distress most likely have viral upper respiratory tract infections and (or) bronchiolitis [1]. The typical presentation of bronchiolitis is a viral infection of the lower respiratory tract often characterized by acute inflammation, edema, increased mucus production, bronchospasm, and clinical presentation of cough, tachypnea, wheezing, crackles, use of accessory muscles, and (or) nasal flaring [2,3]. It is the leading cause of infant hospitalization worldwide and affects more than one-third of children, 0-2 years of age [1]. Despite the common nature of bronchiolitis, and the availability of clinical practice guidelines, there is wide variation in the clinical management, and how it is diagnosed and treated $[1,4,5]$. Utilization of unnecessary investigations and ineffective medication and interventions for the management of bronchiolitis have been costly for the health care system and reflect significant morbidity and burden for families [6, 7]. Often their chest X-rays (CXRs) are misinterpreted as bacterial infections and a detailed respiratory assessment is not performed [1]. As a result, these children may be prescribed pharmacological treatments such as steroids, bronchodilators, and antibiotics, all of which are not recommended treatments of bronchiolitis [1, 2, 8, 9].

Many infants are managed outside children's hospitals where physicians may be less comfortable and familiar with the management of bronchiolitis. To help reduce the amount of unnecessary interventions as well as mitigate clinical anxiety faced by physicians when avoiding these treatments, several strategies have been suggested, including specialized training for ED physicians [10] and use of more detailed and systematic respiratory assessment tools $[8,11]$. The American Academy of Pediatrics (AAP) in their original (2006) and updated (2014) clinical practice guidelines $[2,9]$, as well as the Canadian Paediatric Society (CPS) [1], recommend clinicians diagnose and assess disease severity

${ }^{1}$ Faculty of Health Sciences, University of Ontario Institute of Technology, Oshawa, Canada

${ }^{2}$ Department of Respiratory Therapy, Hospital for Sick Children, Toronto, Canada

${ }^{3}$ Department of Physical Therapy and Rehabilitation Sciences Institute, University of Toronto, Toronto, Canada

${ }^{4}$ Paediatrics, Lakeridge Health, Oshawa, Canada

${ }^{5}$ University of Ontario Institute of Technology-Canadian Memorial Chiropractic College Centre for Disability Prevention and Rehabilitation, Oshawa and Toronto, Canada

Correspondence: Mika L. Nonoyama, Faculty of Health Sciences, University of Ontario Institute of Technology, 2000 Simcoe St North, Oshawa, ON L1H 7K4 Canada. Tel.: 905-721-8668 ext. 5329; Email: mika.nonoyama@uoit.ca

Published online at https://www.cjrt.ca on 15 February 2019

This open-access article is distributed under the terms of the Creative Commons Attribution Non-Commercial License (CC BY-NC) (http:// creativecommons.org/licenses/by-nc/4.0/), which permits reuse, distribution and reproduction of the article, provided that the original work is properly cited and the reuse is restricted to noncommercial purposes. For commercial reuse, contact editor@csrt.com 
based on history and physician examination and avoid routine use of laboratory and radiologic tests for diagnosis. In a prospective cohort study, Schuh et al. [10] found radiography was not necessary for children who present to the ED with a typical presentation of bronchiolitis. Better alternatives to determine severity of illness may include oxygen saturation and respiratory assessment tools. Validated and reliable clinical scoring scales could be easily implemented in the hospital, ED, and outpatient settings [9] and may be particularly useful in smaller community hospitals where paediatric specialists are not readily available [12].

Although steps to standardize care for bronchiolitis have been initiated by the AAP and CPS, uptake has not been widespread. Little is known regarding the management of children with bronchiolitis in EDs in smaller community hospital. The purpose of this study is to describe the characteristics of children admitted to a community-based hospital ED and diagnosed with bronchiolitis and to explore the utility of using signs and symptoms to measure illness severity. We hypothesized that children with bronchiolitis admitted to a community-based hospital would have received treatments not recommended by the guidelines, and those with more severe illness would have worse clinical signs and symptoms and respiratory assessment scores.

\section{MATERIALS AND METHODS}

This retrospective chart review study took place at a 500-bed acute care community-based hospital in Ontario, Canada (Lakeridge Health). This study was approved by the research ethics boards of Lakeridge Health (5 December 2015) and the University of Ontario Institute of Technology (14 January 2016).

Inclusion criteria included children less than 2 years old who met the International Classification of Diseases 10 (ICD10) J21 acute bronchiolitis: J21.0 acute bronchiolitis due to respiratory syncytial virus, J21.1 acute bronchiolitis due to human metapneumovirus, J21.8 acute bronchiolitis due to other specified organisms, and J21.9 acute bronchiolitis, unspecified [13]. Children with chronic lung disease (e.g., asthma, cystic fibrosis), bronchopulmonary dysplasia, congestive heart failure, or immunodeficiency were excluded.

Outcomes included demographic information (e.g., age, gender), admission and discharge date, reason for admission, ED disposition, presenting and ongoing symptoms (e.g., respiratory, vital signs), diagnostic measures (e.g., CXR), first interpretation of CXRs, and interventions (e.g., medication, respiratory therapies). Lakeridge Health does not directly assess the Respiratory Distress Assessment Instrument (RDAI); therefore, total score was calculated using documented assessments of wheezing and retractions (Table 1) $[12,14]$. For example if "moderate supraclavicular retractions" was documented, a score of 2 was assigned for that item. The RDAI has good internal validity [14] and has been used in measuring respiratory distress in young children with bronchiolitis $[9,14]$. It also has excellent inter-observer reliability in the $\operatorname{ED}[5,14]$ and correlates with other measures of respiratory distress [14]. RDAI scores range from 0 to 17 with higher scores indicating more severe distress. In this study, proxies for greater severity of illness were antibiotics and (or) a CXR use and (or) hospital admission.
The medical charts of children who visited Lakeridge Health EDs (site 1, site 2, site 3, and site 4) between 1 January 2014 and 30 September 2015 were identified. Decision Support at Lakeridge Health identified 280 eligible records (approximately 13 per month). One hundred charts (due to time restrictions) were randomly selected via computer program (Microsoft Excel RAND function), and they were stratified by time periods (winter, spring, summer, fall) to account for seasonal variation. Data were extracted from the hospital's electronic medical record system after an initial pilot test and amendment of the Data Extraction Form. RRD extracted data after extensive training.

\section{Data Analysis}

Patient characteristics and variables are described using means and standard deviations (SD), counts, and proportions. Chi-square tests (proportions) and independent $t$-test (continuous variables) were used to compare children who: had a CXR versus those who did not, were provided oral antibiotics versus those who did not, and were admitted to hospital or discharged home; $p<0.05$ was considered significant. All analyses were completed using IBM SPSS Version 24 (IBM Corporation, 2016).

\section{RESULTS}

The mean (SD) age of the 100 children was 10.6 (8.4) months, with $n=$ $41(41 \%)$ females. The mean (SD) gestational age for $n=31$ children was 37.6 weeks ( $n=5$ were $<37$ weeks, $n=69$ not reported). The primary reason for admission was respiratory distress (38\%), cough with or without fever (25\%), and vomiting with or without fever or diarrhea (12\%). Sixty-seven percent had a CXR done, with $51 \%$ initially interpreted by the ED physician and $16 \%$ by a radiologist. Seventeen percent were given oral antibiotics, $65 \%$ inhaled bronchodilators, $19 \%$ oral steroids, and (or) $16 \%$ supplemental oxygen. More children had moderate to severe respiratory distress (43\%), while $23 \%$ had mild distress (34\% were not reported in the chart). The majority of children $(81 \%)$ were discharged home with the remaining admitted (maximum length of stay of 6 days). The mean (SD) total calculated RDAI score was 4.98 (2.43); range 0-11 (Table 2).

There was a significant difference in total calculated RDAI score between those given oral antibiotics (mean (95\% confidence interval), 6.35 (4.96-7.75)) versus not given oral antibiotics (4.70 (4.20-5.20)), $p=0.01$. Those who received a CXR had a significantly higher supplemental oxygen flowrate $(1.4(0.6-2.1)$ litres per minute $(\mathrm{lpm}))$ versus those who did not $(0.15(-0.05$ to 0.35$) \mathrm{lpm}), p=0.002$. There was a significant difference in the physical appearance on admission $(p=0.02)$ and ED disposition ( $p=0.03$ ): more children who did not receive a CXR or were discharged home were "calm and normal" (or not reported) versus the CXR group or those admitted to hospital ( $n=32(97 \%)$ vs. $n=52$ $(78 \%), n=51(63 \%)$ vs. $n=17(90 \%)$, respectively). More children in the CXR group were in abnormal positions (tri-pod position, head bobbing) $(n=15(22 \%))$ versus those who were not $(n=1(3 \%))$. More children who were discharged home had abnormal positions $(n=30(37 \%))$ versus those admitted to hospital $(n=2(10 \%))$. There was no significant

\section{TABLE 1}

The Respiratory Distress Assessment Instrument scoring [14]

\begin{tabular}{|c|c|c|c|c|c|c|}
\hline & \multicolumn{5}{|c|}{ Points } & \multirow[b]{2}{*}{ Maximum points } \\
\hline & 0 & 1 & 2 & 3 & 4 & \\
\hline \multicolumn{7}{|l|}{ Wheezing } \\
\hline Expiration & None & End & $1 / 2$ & $3 / 4$ & All & 4 \\
\hline Inspiration & None & Part & All & - & - & 2 \\
\hline Lung fields & None & $\begin{array}{l}\text { Segmental: } \\
\leq 2 \text { of } 4 \text { lung fields }\end{array}$ & $\begin{array}{l}\text { Diffuse: } \\
\geq 3 \text { of } 4 \text { lung fields }\end{array}$ & - & - & 2 \\
\hline \multicolumn{7}{|l|}{ Retractions } \\
\hline Supraclavicular & None & Mild & Moderate & Marked & - & 3 \\
\hline Intercostal & None & Mild & Moderate & Marked & - & 3 \\
\hline Subcostal & None & Mild & Moderate & Marked & - & 3 \\
\hline
\end{tabular}




\section{TABLE 2}

Patient characteristics of a sample $(n=100 / 280)$ of children with bronchiolitis who did and did not receive a CXR or oral antibiotics or were admitted to hospital or discharged home

\begin{tabular}{|c|c|c|c|c|c|c|c|c|c|c|}
\hline & $\begin{array}{c}\text { Entire } \\
\text { Cohort } \\
(n=100)\end{array}$ & $\begin{array}{c}\text { CXR } \\
(n=67)\end{array}$ & $\begin{array}{l}\text { No CXR } \\
(n=33)\end{array}$ & $p$ & $\begin{array}{l}\text { Oral Antibiotics } \\
\qquad(n=17)\end{array}$ & $\begin{array}{c}\text { No Oral } \\
\text { Antibiotics } \\
(n=83)\end{array}$ & $p$ & $\begin{array}{c}\text { Admitted } \\
(n=19)\end{array}$ & $\begin{array}{l}\text { Discharged } \\
\quad(n=81)\end{array}$ & $p$ \\
\hline \multicolumn{11}{|l|}{ Variable, mean (SD) } \\
\hline Age, months & $10.6(8.4)$ & $10.8(8.6)$ & $10.2(8.1)$ & 0.72 & $14.5(8.7)$ & $9.8(8.1)$ & 0.05 & $6.8(7.2)$ & $11.5(8.4)$ & 0.16 \\
\hline Respiratory rate, ${ }^{a}$ breaths $/ \mathrm{min}$ & $42(12)$ & $42.7(11.3)$ & $39.6(13.8)$ & 0.26 & $41.9(13.8)$ & $41.7(12.0)$ & 0.95 & $44.2(9.3)$ & $41.1(12.8)$ & 0.09 \\
\hline Heart rate ${ }^{a}$ beats $/ \min$ & $151(17)$ & $151(16.7)$ & $151.6(18.1)$ & 0.85 & $148.6(20.1)$ & $151.7(16.5)$ & 0.56 & $153.1(11.9)$ & $150.7(18.2)$ & 0.07 \\
\hline Oxygen saturation, ${ }^{\mathrm{a}} \%$ & $94(3)$ & $94.8(3.5)$ & $95.2(3.6)$ & 0.57 & $94.6(4.1)$ & $95.0(3.4)$ & 0.73 & $95.4(3.5)$ & $94.8(3.5)$ & 0.78 \\
\hline Oxygen flowrate, ${ }^{a}$ litres/min & $1(3)$ & $1.4(3.0)$ & $0.15(0.57)$ & 0.002 & $0.94(2.6)$ & $0.98(2.6)$ & 0.96 & $1.4(2.9)$ & $0.88(2.5)$ & 0.28 \\
\hline RDAl calculated total score & $.98(2.4)$ & $5.01(2.5)$ & $4.91(2.2)$ & 0.83 & $6.35(2.7)$ & $4.70(2.2)$ & 0.03 & $5.05(2.0)$ & $4.96(2.5)$ & 0.19 \\
\hline \multicolumn{11}{|l|}{ Variable, frequency (\%) } \\
\hline Female & $41(41)$ & $26(39)$ & $15(46)$ & 0.52 & $9(53)$ & $32(39)$ & 0.27 & $11(58)$ & $33(40)$ & 0.91 \\
\hline CXR done & $67(67)$ & - & - & - & $13(77)$ & $54(65)$ & 0.36 & $12(63)$ & $45(68)$ & 0.69 \\
\hline Oral antibiotics & $17(17)$ & $13(19)$ & $4(12)$ & 0.36 & - & - & - & $2(11)$ & $15(19)$ & 0.40 \\
\hline IV antibiotics & $1(1)$ & $1(2)$ & 0 & 0.48 & $1(6)$ & 0 & 0.03 & 0 & $1(1)$ & 0.63 \\
\hline Inhaled medication & $65(65)$ & $42(63)$ & $23(70)$ & 0.49 & $9(53)$ & $56(68)$ & 0.25 & $13(68)$ & $52(64)$ & 0.73 \\
\hline Oral steroids & $19(19)$ & $12(18)$ & $7(21)$ & 0.69 & $4(24)$ & $15(18)$ & 0.60 & $2(11)$ & $17(21)$ & 0.30 \\
\hline Supplemental oxygen & $16(16)$ & $13(19)$ & $3(9)$ & 0.19 & $3(18)$ & $13(16)$ & 0.84 & $5(26)$ & $11(14)$ & 0.17 \\
\hline \multicolumn{11}{|l|}{ ED disposition } \\
\hline Home & $81(81)$ & $55(82)$ & $26(79)$ & 0.69 & $15(88)$ & $66(80)$ & 0.40 & - & - & - \\
\hline Inpatient & 19 (19) & $12(18)$ & $7(21)$ & & $2(12)$ & $17(21)$ & & - & - & - \\
\hline \multicolumn{11}{|l|}{ CXR interpretation } \\
\hline ED physician & $51(51)$ & $48(72)$ & - & - & $10(59)$ & $41(49)$ & 0.71 & $12(63)$ & $39(48)$ & 0.21 \\
\hline Radiologist & $16(16)$ & $14(21)$ & - & & 3 (18) & $13(16)$ & & (0) & $16(20)$ & \\
\hline Paediatrician & $4(4)$ & $4(6)$ & - & & $1(6)$ & $3(4)$ & & $1(5)$ & $3(4)$ & \\
\hline Not reported & $29(29)$ & $1(2)$ & - & & $3(18)$ & $26(31)$ & & $6(32)$ & $23(28)$ & \\
\hline \multicolumn{11}{|l|}{ Reason for ED admission } \\
\hline Respiratory distress & $38(38)$ & $25(37)$ & $13(39)$ & 0.10 & $3(18)$ & $35(42)$ & 0.20 & $12(63)$ & $26(32)$ & 0.31 \\
\hline Cough \pm fever & $25(25)$ & $19(28)$ & $6(18)$ & & $4(24)$ & $21(25)$ & & $3(17)$ & $22(27)$ & \\
\hline Vomit \pm fever or diarrhea & $12(12)$ & $5(8)$ & $4(12)$ & & $1(6)$ & $8(10)$ & & $1(5)$ & $11(14)$ & \\
\hline Fever & $10(10)$ & $9(13)$ & $1(3)$ & & $4(24)$ & $6(7)$ & & $1(5)$ & $9(11)$ & \\
\hline Cold symptoms & $9(9)$ & $6(9)$ & $3(9)$ & & $2(12)$ & $7(8)$ & & $1(5)$ & $8(10)$ & \\
\hline Other & $6(6)$ & $3(5)$ & $3(9)$ & & $2(12)$ & $4(5)$ & & $1(5)$ & $5(6)$ & \\
\hline \multicolumn{11}{|l|}{ Physical appearance $^{a}$} \\
\hline Calm and normal & & & & & & & & & & \\
\hline (and not reported) & $84(84)$ & $52(78)$ & $32(97)$ & 0.02 & $9(53)$ & $59(71)$ & 0.16 & $17(90)$ & $51(63)$ & 0.03 \\
\hline Abnormal $^{\mathrm{b}}$ & $16(16)$ & $15(22)$ & $1(3)$ & & $8(48)$ & $24(29)$ & & 2 (10) & $30(37)$ & \\
\hline
\end{tabular}

${ }^{a}$ On admission to the ED.

${ }^{b}$ Includes tri-pod position and head bobbing.

Note: Bold values are statistically signficant. CXR, chest X-ray; RDAI, Respiratory Distress Assessment Instrument; ED, emergency department; IV, intravenous.

difference in any other variable for those who did or did not have a CXR, provided or not provided oral antibiotics, or hospitalized versus discharged home (Table 2).

\section{DISCUSSION}

This study describes the management of children with bronchiolitis admitted to a community-based ED. The majority (67\%) of children admitted had a CXR, $65 \%$ bronchodilators, $19 \%$ oral steroids, and $17 \%$ oral antibiotics. Children who had a CXR had significantly higher supplemental oxygen flowrates and worse physical appearance on admission. Children who received oral antibiotics had a significantly higher RDAI score (greater respiratory distress) compared with those who did not.

Both the AAP and CPS bronchiolitis guidelines do not recommend the routine use of radiological investigations or bronchodilators and steroids $[2,11]$. Our study showed a high number of children had a CXR $(67 \%)$, though this is similar to other studies $[15,16]$ varying between $49 \%$ and $75 \%$, even after the release of the clinical practice guidelines [4, 15, 17-20]. Prior studies have shown patients admitted to a general ED have higher odds of receiving a CXR compared with a specialized children's facility. This is likely because specialized children's facilities would have more expertise in the management of bronchiolitis [16].

Our study also found $65 \%$ of children received bronchodilators and $19 \%$ steroids. In a Canadian survey, $67 \%$ of paediatric ED physicians indicated they "typically" provide bronchodilators (Salbutamol or Epinephrine) and 3\% "always" and 73\% "sometimes" provide steroids [21]. In a follow-up retrospective study, the same research group found these numbers were even higher in community hospitals; ED physicians prescribed bronchodilators and steroids $80 \%$ and $31 \%$ of the time, respectively [19].

CXR and bronchodilator use may have been high in our study because adult ED physicians, who likely had less experience with bronchiolitis, were the first health care provider to assess the children. CXRs may have been ordered based on presenting clinical findings and (or) to rule out pneumonia. Our results showed children who had a CXR had significantly worse physical appearance and oxygen flowrates compared with those who did not have a CXR. With respect to bronchodilators, general paediatricians tend to prescribe these medications more often compared with paediatric pulmonologists [22]. At Lakeridge Health and many other health centres, paediatric pulmonologists are not involved with bronchiolitis management compared with generalists like adult ED physicians. Regardless of expertise and location, our study and past literature show a high percentage of unnecessary bronchodilator and steroid prescriptions. These results may indicate the need for processes to help increase education and awareness of bronchiolitis management for both specialized centres and community-based hospital generalists [23].

Despite the high use of CXR and bronchodilators, our study showed fewer children $(17 \%)$ were prescribed oral antibiotics. The AAP 
recommends against their use for bronchiolitis [2,9], and other studies have shown higher numbers ranging from $30 \%$ to $73 \%[8,11,15$, $18,24]$. Additionally, despite the high number of children receiving a CXR, there was similar antibiotic use between groups. Among the $67 \%$ of patients that received a CXR, only $19 \%$ of them received oral antibiotics (similar to those who did not have a CXR (12\%)). This is in contrast to previous studies that found in bronchiolitis CXR is associated with antibiotics use [4], and when ED physicians interpreted CXRs, antibiotic use was $>5$ times post-radiography compared with pre-radiography [9].

Our study also showed the number of patients admitted to the hospital (19\%) was lower compared with other studies (mix of communitybased and paediatric centres), which reported between $30 \%$ and $40 \%$ $[19,25,26]$. Low admissions may be attributed to our cohort having less severe bronchiolitis. First, more children admitted to hospital had a "calm and normal" physical appearance $(n=30)$ compared with those discharged home $(n=2)$. In addition the mean (SD) oxygen saturation in our study $(94(3 \%))$ was well above the accepted safe and clinically effective threshold of $90 \%$ [27]. Studies have shown that in bronchiolitis, lower oxygen saturation is a predictor of hospital admission and length of stay, especially when it is less than $90 \%[27,28]$.

The AAP bronchiolitis clinical practice guidelines recommend that diagnosis be based on history and physical exam, and laboratory and radiological studies should not be routinely ordered [2, 11]. Although this study did not investigate different methods of diagnoses, it did explore alternative measures of severity as proxies for laboratory and radiological tests. Our results support our assumptions that children receiving antibiotics and (or) CXR have more severe illness. We found children who had a CXR had significantly worse physical appearance (tri-pod position, head bobbing), and oxygen flowrates (1.4 (0.6-2.1) lpm) compared with those who did not $(0.15$ (-0.05 to $0.35) \mathrm{lpm}, p=0.002)$. Those children who received antibiotics had significantly worse RDAI scores (mean (95\% CI), 6.35 (4.96-7.75)) versus those who did not $(4.70(4.20-5.20)), p=0.01$. These findings may support physical investigations as "stand-alone" assessments of illness severity, making the CXR and other laboratory tests redundant. This may guide clinicians toward accurate diagnosis and appropriate management.

Utilizing AAP or other clinical practice guidelines [2, 9, 23] may help hospitals optimize the management of children with bronchiolitis. Previous studies have shown these guidelines have decreased the use of diagnostics (CXR) and medications (bronchodilators, steroids, antibiotics) for children with bronchiolitis $[15,16,18,23]$. This is especially important for smaller community-based EDs where physicians may not have the opportunity to see a large number of children with bronchiolitis. Our study (and previous literature) shows low uptake of CPS bronchiolitis recommendations and highlights the challenges in changing clinical behaviour and culture, especially in a smaller community hospital. Development of institutional or health region specific clinical practice guidelines or protocols (based on CPS) that includes training and education is required to improve diagnosis and treatment of bronchiolitis in this setting. These specific guidelines have been effective in changing local practice of bronchiolitis management, especially if developed with all relevant stakeholders and using thoughtful and rigorous methods. Touzet et al. [29] reported on the impact of a bronchiolitis guideline developed during a consensus conference of primary health care physicians. It was disseminated to general and specialty paediatricians via traditional and medical media, mailings, and on a web site. There was modest improvement in bronchodilator and steroid usage (7\%-14\%, $p=0.04)$ but no change in avoiding use of CXR or antibiotics. After the implementation of local guidelines, a Swiss study [22] demonstrated a large reduction in the use of bronchodilators, antibiotics, and steroids for both outpatients and inpatients. For outpatients, bronchodilator prescriptions dropped from $60 \%$ to $23 \%$ and steroids from $34 \%$ to $6 \%$ [22]. Greater success in guideline utilization (compared with Touzet et al [29]) may have been attributed to its development in collaboration with paediatric physicians using a multi-step comprehensive approach. In addition they were disseminated in several medical and scientific journals, at key conferences, at local teaching rounds, and via leaflets [22].
In a community-based ED, this approach may help optimize the most up-to-date and evidence-informed diagnosis and treatments of bronchiolitis if it includes collaborations with both community hospital clinicians and paediatric experts.

\section{Limitations}

This study has several limitations. First were limitations common to retrospective study designs [30]. Data were from a single regional center in Ontario, Canada, and involved a small sample size of 100 patients of mild to moderate severity. Statistical analyses were completed with no adjustment for multiple comparisons. Some variables (ED disposition, CXR interpretation, physical appearance, gestational age) were not reported in patient charts and may have influenced the results if available. The RDAI was not directly assessed but calculated based on retrospective clinician assessment of wheezing and retractions (and not validated). Physical appearance (tri-pod position, head bobbing) was used as a proxy of disease severity. We acknowledge this is not a typical bronchiolitis sign and may have been an indication of other diagnoses. We did not include other variables associated with bronchiolitis such as patient's overall health condition, presence of dehydration, response to the treatment, and socioeconomic status. Because of the retrospective nature, we were unable to evaluate direct physician behaviour and their rationale for resource utilization. We did not include a comparison group of children without bronchiolitis or compare children included with those excluded from the study. Diagnosis of bronchiolitis was identified based on electronic medical records according to ICD-10 code [13]. The data entered were dependent on physicians' diagnosis in the ED and may not have captured all patients with bronchiolitis. Based on the diagnostic codes we did thorough chart reviews for children that met our eligibility criteria.

\section{CONCLUSIONS}

A large number of children admitted to a community-based ED for bronchiolitis may have received unnecessary CXR, bronchodilators, and steroids. Assessing physical and respiratory distress may be more effective at determining illness severity compared with radiological and (or) laboratory testing. Development of institutional or health region specific clinical practice guidelines that considers local culture and includes collaboration with all stakeholders may aid in the optimal diagnosis and treatment of bronchiolitis for community-based EDs.

\section{REFERENCES}

1. Friedman JN, Rieder MJ, Walton JM. Bronchiolitis: Recommendations for diagnosis, monitoring and management of children one to 24 months of age. Paediatrics Child Health 2014;19(9):485-98. doi: 10.1093/ $\mathrm{pch} / 19.9 .485$.

2. American Academy of Pediatrics Subcommittee on Diagnosis and Management of Bronchiolitis. Diagnosis and management of bronchiolitis. Pediatrics 2006;118(4):1774-93. doi: 10.1542/peds.2006-2223.

3. Carroll KN, Gebretsadik T, Griffin MR, et al. Increasing burden and risk factors for bronchiolitis-related medical visits in infants enrolled in a state health care insurance plan. Pediatrics 2008;122(1):58-64. doi: 10.1542/peds.2007-2087.

4. Christakis DA, Cowan CA, Garrison MM, Molteni R, Marcuse E, Zerr DM. Variation in inpatient diagnostic testing and management of bronchiolitis. Pediatrics 2005;115(4):878-84. doi: 10.1542/peds.2004-1299.

5. Wang EE, Law BJ, Boucher FD, et al. Pediatric Investigators Collaborative Network on Infections in Canada (PICNIC) study of admission and management variation in patients hospitalized with respiratory syncytial viral lower respiratory tract infection. J Pediatr 1996;129(3):390-5. doi: 10.1016/S0022-3476(96)70071-9.

6. Leader S, Yang H, DeVincenzo J, Jacobson P, Marcin JP, Murray DL. Time and out-of-pocket costs associated with respiratory syncytial virus hospitalization of infants. Value Health 2003;6(2):100-6. doi: 10.1046/j.1524-4733.2003.00220.x.

7. Stang P, Brandenburg N, Carter B. The economic burden of respiratory syncytial virus-associated bronchiolitis hospitalizations. Arch Pediatr Adolesc Med 2001;155(1):95-6. doi: 10.1001/archpedi.155.1.95.

8. Farley R, Spurling GK, Eriksson L, Del Mar CB. Antibiotics for bronchiolitis in children under two years of age. Cochrane Database Syst Rev 2014;10):Cd005189. doi: 10.1002/14651858.CD005189.pub4. 
9. Ralston SL, Lieberthal AS, Meissner HC, et al. Clinical practice guideline: The diagnosis, management, and prevention of bronchiolitis. Pediatrics 2014;134(5):e1474-502. doi: 10.1542/peds.2014-2742.

10. Schuh S, Lalani A, Allen U, et al. Evaluation of the utility of radiography in acute bronchiolitis. J Pediatr 2007;150(4):429-33. doi: 10.1016/j. jpeds.2007.01.005.

11. De Brasi D, Pannuti F, Antonelli F, de Seta F, Siani P, de Seta L. Therapeutic approach to bronchiolitis: Why pediatricians continue to overprescribe drugs? Italian J Pediatr 2010;36:67. doi: 10.1186/1824-7288-36-67.

12. Destino L, Weisgerber MC, Soung P, et al. Validity of respiratory scores in bronchiolitis. Hosp Pediatr 2012;2(4): 202-9. doi: 10.1542/ hpeds.2012-0013.

13. World Health Organization. International Statistical Classification of Diseases and Related Health Problems 10th Revision. Geneva: World Health Organization; 2016.

14. Lowell DI, Lister G, Von Koss H, McCarthy P. Wheezing in infants: The response to epinephrine. Pediatrics 1987;79(6):939-45.

15. Johnson LW, Robles J, Hudgins A, Osburn S, Martin D, Thompson A. Management of bronchiolitis in the emergency department: Impact of evidence-based guidelines? Pediatrics 2013;131:Suppl 1S103-9. doi: $10.1542 /$ peds.2012-1427m.

16. McCulloh RJ, Smitherman SE, Koehn KL, Alverson BK. Assessing the impact of national guidelines on the management of children hospitalized for acute bronchiolitis. Pediatric Pulmonol 2014;49(7):688-94. doi: 10.1002/ppul.22835.

17. Knapp JF, Simon SD, Sharma V. Quality of care for common pediatric respiratory illnesses in United States emergency departments: Analysis of 2005 National Hospital Ambulatory Medical Care Survey Data. Pediatrics 2008;122(6):1165-70. doi: 10.1542/peds.2007-3237.

18. Parikh K, Hall M, Teach SJ. Bronchiolitis management before and after the AAP guidelines. Pediatrics 2014;133(1):e1-7. doi: 10.1542/ peds.2013-2005.

19. Plint AC, Taljaard M, McGahern C, et al. Management of Bronchiolitis in Community Hospitals in Ontario: A Multicentre Cohort Study. Cjem 2016;18(6):443-52. doi: 10.1017/cem.2016.7.

20. Todd J, Bertoch D, Dolan S. Use of a large national database for comparative evaluation of the effect of a bronchiolitis/viral pneumonia clinical care guideline on patient outcome and resource utilization. Arch Pediatr Adolesc Med 2002;156(11):1086-90. doi: 10.1001/ archpedi.156.11.1086.

21. Plint AC, Grenon R, Klassen TP, Johnson DW. Bronchodilator and steroid use for the management of bronchiolitis in Canadian pediatric emergency departments. Cjem 2015;17(1):46-53. doi: 10.2310/8000. 2013.131325.

22. Barben J, Kuehni CE, Trachsel D, Hammer J. Management of acute bronchiolitis: Can evidence based guidelines alter clinical practice? Thorax 2008;63(12):1103-9. doi: 10.1136/thx.2007.094706.

23. Breakell R, Thorndyke B, Clennett J, Harkensee C. Reducing unnecessary chest X-rays, antibiotics and bronchodilators through implementation of the NICE bronchiolitis guideline. Eur J Pediatr 2018;177(1):47-51.

24. Dallas A, Magin P, Morgan S, et al. Antibiotic prescribing for respiratory infections: A cross-sectional analysis of the ReCEnT study exploring the habits of early-career doctors in primary care. Fam Pract 2015;32(1):49-55. doi: 10.1093/fampra/cmu069.

25. Corneli HM, Zorc JJ, Holubkov R, et al. Bronchiolitis: Clinical characteristics associated with hospitalization and length of stay. Pediatric Emerg Care 2012;28(2):99-103. doi: 10.1097/PEC.0b013e3182440b9b.

26. Marlais M, Evans J, Abrahamson E. Clinical predictors of admission in infants with acute bronchiolitis. Arch Dis Child 2011;96(7):648-52. doi: 10.1136/adc.2010.201079.

27. Cunningham S, Rodriguez A, Adams T, et al. Oxygen saturation targets in infants with bronchiolitis (BIDS): A double-blind, randomised, equivalence trial. Lancet (London, England) 2015;386(9998):1041-8. doi: 10.1016/S0140-6736(15)00163-4.

28. Voets S, van Berlaer G, Hachimi-Idrissi S. Clinical predictors of the severity of bronchiolitis. European J Emerg Med 2006;13(3):134-8. doi: 10.1097/01.mej.0000206194.85072.33.

29. Touzet S, Refabert L, Letrilliart L, Ortolan B, Colin C. Impact of consensus development conference guidelines on primary care of bronchiolitis: Are national guidelines being followed? J Eval Clin Pract 2007;13(4):651-6. doi: 10.1111/j.1365-2753.2007.00781.x.

30. Hulley SB, Cummings SR, Newman TB. Chapter 7. Designing crosssectional and cohort studies. In: Designing Clinical Research. 4th ed: Lippincott Williams \& Wilkins, Philadelphia, PA; 2013. p. 85-95. 